Published on Reviews in History (https://reviews.history.ac.uk)

\title{
INTERVIEW: Daniel Snowman talks to Margaret MacMillan
}

Review Number: 2080

Publish date: Tuesday, 14 March, 2017

Author: Margaret MacMillan

ISBN: 9781781255124

Date of Publication: 2016

Price: $£ 11.99$

Pages: 288pp.

Publisher: Profile Books

Publisher url: http://www.margaretmacmillan.com/

Place of Publication: London

Reviewer: Margaret MacMillan

In the latest of our occasional Reviews in History podcast series, Daniel Snowman talks to Margaret MacMillan about her background, career, key publications and future plans.

Margaret MacMillan is Professor of International History at the University of Oxford.

Daniel Snowman is a writer, lecturer and broadcaster on social and cultural history.

You can listen to the interview here [2].

\section{Other reviews:}

Guardian

https://www.theguardian.com/books/2016/feb/10/historys-people-margaret-macmillan-review [3]

Independent

http://www.independent.co.uk/arts-entertainment/books/reviews/historys-people-personalities-and-the-pastby-margaret-macmillan-book-review-a6867356.html [4]

Times Higher Education

https://www.timeshighereducation.com/books/review-historys-people-margaret-macmillan-profile-books [5]

Financial Times

https://www.ft.com/content/aab46a88-dbbd-11e5-a72f-1e7744c66818 [6]

Globe and Mail

http://www.theglobeandmail.com/arts/books-and-media/book-reviews/review-margaret-macmillans-historyspeople-examines-those-who-have-made-and-unmade-our-world/article26633446/ [7]

Washington Times

http://www.washingtontimes.com/news/2016/may/1/book-review-historys-people-personalities-and-the-/ [8]

Kirkus Reviews

https://www.kirkusreviews.com/book-reviews/margaret-macmillan/historys-people/ [9]

Source URL:https://reviews.history.ac.uk/review/2080

\section{Links}

[1] https://reviews.history.ac.uk/item/267701

[2] http://podcast.ulcc.ac.uk/accounts/SAScasts/ReviewsinHistory/SnowmanonMacmillancropped.mp3 
[3] https://www.theguardian.com/books/2016/feb/10/historys-people-margaret-macmillan-review

[4] http://www.independent.co.uk/arts-entertainment/books/reviews/historys-people-personalities-and-thepast-by-margaret-macmillan-book-review-a6867356.html

[5] https://www.timeshighereducation.com/books/review-historys-people-margaret-macmillan-profile-books

[6] https://www.ft.com/content/aab46a88-dbbd-11e5-a72f-1e7744c66818

[7] http://www.theglobeandmail.com/arts/books-and-media/book-reviews/review-margaret-macmillanshistorys-people-examines-those-who-have-made-and-unmade-our-world/article26633446/

[8] http://www.washingtontimes.com/news/2016/may/1/book-review-historys-people-personalities-and-the-/

[9] https://www.kirkusreviews.com/book-reviews/margaret-macmillan/historys-people/ 